\title{
On uncertainty relations and entanglement detection with mutually unbiased measurements*
}

\author{
Alexey E. Rastegin \\ Department of Theoretical Physics, Irkutsk State University, Gagarin Bv. 20, Irkutsk 664003, Russia
}

\begin{abstract}
We formulate some properties of a set of several mutually unbiased measurements. These properties are used for deriving entropic uncertainty relations. Applications of mutually unbiased measurements in entanglement detection are also revisited. First, we estimate from above the sum of the indices of coincidence for several mutually unbiased measurements. Further, we derive entropic uncertainty relations in terms of the Rényi and Tsallis entropies. Both the state-dependent and state-independent formulations are obtained. Using the two sets of local mutually unbiased measurements, a method of entanglement detection in bipartite finite-dimensional systems may be realized. A certain trade-off between a sensitivity of the scheme and its experimental complexity is discussed.
\end{abstract}

PACS numbers: 03.65.Ta, 03.67.-a, 03.67.Ud

Keywords: mutually unbiased measurements, generalized entropies, uncertainty principle, bipartite systems, separable states

\section{INTRODUCTION}

Complementarity and entanglement are basic concepts of quantum theory. Heisenberg's uncertainty principle [1] is one of the most known restrictions imposed in the quantum world. A truly non-classical character of entanglement was emphasized in the Schrödinger "cat paradox" paper [2]. Today, quantum properties are considered as powerful resources for potential usage in communication and computation tasks [3]. Historically, uncertainty relations were focused on pairs of canonically conjugate variables $[4,5]$. Recent researches have shown that uncertainty relations give a useful tool for studying complementarity aspects [6]. Quantum entanglement is used as a basic tool in quantum parallelism, quantum cryptography, quantum dense coding, and quantum teleportation [7].

The notion of mutually unbiased bases has much many links with recent studies of quantum information protocols (see, e.g., the review [8] and references therein). Mutually unbiased bases are also an interesting mathematical subject [8]. For example, the problem of maximal set of mutually unbiased bases is still open. In general, the maximal number of MUBs in $d$ dimensions is still an open question [8]. When $d$ is a prime power, the answer $d+1$ is known [9]. For other $d$, i.e., for composite numbers, we only know that the maximal number of MUBs does not exceed $d+1$. The author of [10] proposed the concept of mutually unbiased measurements. The principal result is that a complete set of $d+1$ mutually unbiased measurements has been built explicitly for arbitrary finite $d$ [10]. Hence, we have come across different questions concerning a possible usage of such measurements in quantum information science.

The aim of the present work is to study mutually unbiased measurements in some important respects. The paper is organized as follows. In section II, preliminary facts are reviewed. We also prove an interesting relation between Rényi's entropies of three different orders. In Section III, we derive an upper bound on the sum of the indices of coincidence for a set of mutually unbiased measurements. In Section IV, uncertainty relations for an arbitrary number of such measurements are derived in terms of Rényi's and Tsallis' entropies. Both the state-dependent and stateindependent formulations are given. Applications of mutually unbiased measurements in entanglement detection are examined in Section V. We will see an evidence for trade-off between a sensitivity of the scheme and costs for its implementation. In Section V, we conclude the paper with a summary of results.

\section{PRELIMINARIES}

In this section, we review the required material. First, some notation for spaces and operators is introduced. We then recall the definition of mutually unbiased measurements proposed in [10]. Further, we discuss the Rényi and Tsallis entropies which will be used as measures of uncertainties. We also prove a relation between Rényi's entropies of three different orders. 
Due to sensitivity of quantum states, a measurement stage is one of central questions in quantum protocols [3]. Hence, some selected types of measurements are of special interest in quantum information processing. Mutually unbiased bases are used in quantum state reconstruction [9], quantum error correction [11, 12], detection of quantum entanglement [13], and the mean king's problem [14, 15]. For arbitrary $d$, however, constructing a maximal set of mutually unbiased bases is an open problem. One may try to fit "unbiasedness" with weaker conditions [10]. In this way, we will deal with mutually unbiased measurements.

Let $\mathcal{L}(\mathcal{H})$ be the space of linear operators on $d$-dimensional Hilbert space $\mathcal{H}$. By $\mathcal{L}_{+}(\mathcal{H})$, we denote the set of positive semi-definite operators on $\mathcal{H}$. A density operator $\boldsymbol{\rho} \in \mathcal{L}_{+}(\mathcal{H})$ is normalized by $\operatorname{Tr}(\boldsymbol{\rho})=1$. For operators $\mathrm{X}, \mathrm{Y} \in \mathcal{L}(\mathcal{H})$, their Hilbert-Schmidt inner product is written as [16]

$$
\langle\mathrm{X}, \mathrm{Y}\rangle_{\mathrm{hs}}:=\operatorname{Tr}\left(\mathrm{X}^{\dagger} \mathrm{Y}\right)
$$

Let $\mathcal{A}=\left\{\left|\phi_{n}\right\rangle\right\}_{n=1}^{d}$ and $\mathcal{B}=\left\{\left|\varphi_{n}\right\rangle\right\}_{n=1}^{d}$ be orthonormal bases in $d$ dimensions. They are said to be mutually unbiased, when

$$
\left|\left\langle\phi_{m} \mid \varphi_{n}\right\rangle\right|=\frac{1}{\sqrt{d}},
$$

for all $m, n=1, \ldots, d$. The set $\mathbb{B}=\left\{\mathcal{B}^{(1)}, \ldots, \mathcal{B}^{(M)}\right\}$ is a set of mutually unbiased bases (MUBs), when each two bases from this set are mutually unbiased. When dimensionality $d$ is not a prime power, we do not know the maximal number of MUBs that can be constructed. The answer is not known even for $d=6$ [8]. In some respects, mutually unbiased bases are connected with symmetric informationally complete measurements, shortly SIC-POVMs [8]. Such measurements are also not easy to construct.

The authors of [10] proposed a concept of mutually unbiased measurements (MUMs). Let $\mathcal{P}=\left\{\mathrm{P}_{n}\right\}$ and $\mathcal{Q}=\left\{\mathrm{Q}_{n}\right\}$ be two POVM measurements, each with $d$ elements. We assume that POVM elements satisfy

$$
\begin{aligned}
& \operatorname{Tr}\left(\mathrm{P}_{n}\right)=\operatorname{Tr}\left(\mathrm{Q}_{n}\right)=1, \\
& \operatorname{Tr}\left(\mathrm{P}_{m} \mathrm{Q}_{n}\right)=\frac{1}{d} .
\end{aligned}
$$

The following fact follows from the assumptions. The Hilbert-Schmidt product of two elements from the same POVM can be described in terms of a single parameter $\varkappa[10]$ :

$$
\operatorname{Tr}\left(\mathrm{P}_{m} \mathrm{P}_{n}\right)=\delta_{m n} \varkappa+\left(1-\delta_{m n}\right) \frac{1-\varkappa}{d-1}
$$

General bounds on the parameter $\varkappa$ are written as $1 / d \leq \varkappa \leq 1[10]$. The set $\mathbb{P}=\left\{\mathcal{P}^{(1)}, \ldots, \mathcal{P}^{(M)}\right\}$ is a set of MUMs of the efficiency $\varkappa$, when each two measurements obey the above properties. It turns out that we can reach the aim to build a complete set of $d+1$ mutually unbiased measurements in $d$ dimensions [10].

Let us consider $d^{2}-1$ operators that form an orthogonal basis in the space of traceless Hermitian operators. For instance, we can start with the generators of $\mathrm{SU}(d)$ [10]. Using such operators, one can built a family of traceless Hermitian operators $\mathrm{F}_{n}^{(b)}$ with labels $b=1, \ldots, d+1$ and $n=1, \ldots, d$. The constructed operators obey the condition

$$
\operatorname{Tr}\left(\mathrm{F}_{m}^{(a)} \mathrm{F}_{n}^{(b)}\right)=0 \quad(a \neq b)
$$

For one and the same label $b$, they also satisfy [10]

$$
\operatorname{Tr}\left(\mathrm{F}_{m}^{(b)} \mathrm{F}_{n}^{(b)}\right)=(1+\sqrt{d})^{2}\left[\delta_{m n}(d-1)-\left(1-\delta_{m n}\right)\right],
$$

where $\delta_{m n}$ is the Kronecker symbol.

An explicit construction of MUMs is written as follows [10]. For $b=1, \ldots, d+1$ and $n=1, \ldots, d$, we introduce operators

$$
\mathrm{P}_{n}^{(b)}=\frac{\mathbb{1}}{d}+t \mathrm{~F}_{n}^{(b)}
$$

where $t$ is some parameter that should be chosen. The least quantities among eigenvalues of the operators $\mathrm{F}_{n}^{(b)}$ determine an interval, in which $t$ can be varied [10]. This interval should be such that $\mathrm{P}_{n}^{(b)} \in \mathcal{L}_{+}(\mathcal{H})$ for all values of the labels. With the given $t$, the efficiency parameter is calculated as

$$
\varkappa=\frac{1}{d}+t^{2}(1+\sqrt{d})^{2}(d-1) .
$$


The range of $t$ leads to the corresponding range of $\varkappa$. The measurements $\mathcal{P}^{(b)}=\left\{\mathrm{P}_{n}^{(b)}\right\}_{n=1}^{d}$ then form a complete set of MUMs of the efficiency (9).

As measures of an uncertainty in quantum measurements, we will use the Rényi and Tsallis entropies. The concept of entropy is of great importance in both information theory and statistical physics. In addition to the Shannon entropy, other entropic measures were found to be useful. The Rényi and Tsallis entropies are both very important [17]. For the given probability distribution, its Rényi $\alpha$-entropy is defined as [18]

$$
R_{\alpha}(p):=\frac{1}{1-\alpha} \ln \left(\sum_{n} p_{n}^{\alpha}\right)
$$

where $\alpha>0$ and $\alpha \neq 1$. This quantity is a non-increasing function of $\alpha$ [18]. Other properties of parametric dependence of (10) are discussed in [19]. The Renyi entropy of order $\alpha=2$ is also known as the collision entropy $[6,17]$. It is written as

$$
R_{2}(p)=-\ln \left(\sum_{n} p_{n}^{2}\right)
$$

In the limit $\alpha \rightarrow \infty$, we have the so-called min-entropy

$$
R_{\infty}(p)=-\ln \left(\max p_{n}\right) .
$$

The min-entropy is of specific interest in cryptography [20]. It is also linked with the extrema of the discrete Wigner function [21]. Uncertainty bounds on Rényi's entropies are significant in studying the connection between complementarity and uncertainty principles [22]. Using the Rényi entropy, the writers of [23] obtained trade-off relations for a trace-preserving quantum operation. An extension of such trade-off relations in terms of the unified entropies was given in [24].

The notion of Tsallis entropy is widely used in non-extensive statistical mechanics [25]. The non-extensive entropy of positive degree $\alpha \neq 1$ is defined as [26]

$$
H_{\alpha}(p):=\frac{1}{1-\alpha}\left(\sum_{n} p_{n}^{\alpha}-1\right)
$$

With the factor $\left(2^{1-\alpha}-1\right)^{-1}$ instead of $(1-\alpha)^{-1}$, this function was deduced by Havrda and Charvát [27]. The entropy (13) is concave for all $\alpha>0$. In more detail, properties of the entropy (13) and related functionals are considered in $[28,29]$. It is convenient to rewrite (13) as

$$
H_{\alpha}(p)=-\sum_{n} p_{n}^{\alpha} \ln _{\alpha}\left(p_{n}\right)=\sum_{n} p_{n} \ln _{\alpha}\left(\frac{1}{p_{n}}\right) .
$$

Here, we used the $\alpha$-logarithm defined for $\alpha>0 \neq 1$ and $x>0$ as

$$
\ln _{\alpha}(x)=\frac{x^{1-\alpha}-1}{1-\alpha}
$$

In the limit $\alpha \rightarrow 1$, we obtain $\ln _{\alpha}(x) \rightarrow \ln x$ and the standard Shannon entropy

$$
H_{1}(p)=-\sum_{n} p_{n} \ln p_{n}
$$

Of course, the right-hand side of (10) also gives (16) in this limit. Various applications of the above entropies and their quantum counterparts are discussed in the book [17].

Analyzing the case of detection inefficiencies, we will use the method of $[30,31]$. To the given value $\eta \in[0 ; 1]$ and probability distribution $\left\{p_{n}\right\}$, one assigns a "distorted" distribution:

$$
p_{n}^{(\eta)}=\eta p_{n}, \quad p_{\varnothing}^{(\eta)}=1-\eta
$$

Here, the parameter $\eta \in[0 ; 1]$ describes a detector efficiency. By $p_{\varnothing}^{(\eta)}$, we denote the probability of the no-click event. The described model of distorted probabilities was introduced in studying entropic Bell inequalities with detector inefficiencies [32]. Further development of Bell inequalities with detection inefficiencies was given in [33]. As was shown in the paper [33], for all $\alpha>0$ we have

$$
H_{\alpha}\left(p^{(\eta)}\right)=\eta^{\alpha} H_{\alpha}(p)+h_{\alpha}(\eta)
$$


where $H_{\alpha}\left(p^{(\eta)}\right)$ denotes the entropy of "distorted" distribution (17). As usual, the binary Tsallis entropy $h_{\alpha}(\eta)$ is written as

$$
h_{\alpha}(\eta):=-\eta^{\alpha} \ln _{\alpha}(\eta)-(1-\eta)^{\alpha} \ln _{\alpha}(1-\eta) .
$$

Entropic uncertainty relations with detection inefficiencies were derived for mutually unbiased bases in [30] and for a general SIC-POVM in [31]. We will apply this method to mutually unbiased measurements.

Deriving uncertainty relations in terms of the Rényi entropies, we will deal with the following situation. Both the collision entropy and min-entropy can be calculated or estimated from below. We wish to obtain a lower bound on the Rényi entropy of order $\alpha \geq 2$. An answer to the question is written as follows.

Proposition 1 For $\alpha \in[2 ; \infty]$, the Rényi $\alpha$-entropy is bounded from below as

$$
R_{\alpha}(p) \geq \frac{1}{\alpha-1} R_{2}(p)+\frac{\alpha-2}{\alpha-1} R_{\infty}(p) .
$$

Proof. We will deal with finite $\alpha \geq 2$. We first write the inequality

$$
\sum_{n} p_{n}^{\alpha} \leq\left(\max p_{n}\right)^{\alpha-2} \sum_{n} p_{n}^{2}
$$

The function $x \mapsto(1-\alpha)^{-1} \ln x$ decreases for $\alpha \geq 2$. Combining this fact with the formulas (10), (11), and (12) completes the proof.

It should be pointed out that, for $\alpha \in[2 ; \infty]$, the Rényi $\alpha$-entropy can be estimated in terms of only the collision entropy. As was mentioned in [30], for $\alpha \geq 2$ we have

$$
R_{\alpha}(p) \geq \frac{\alpha}{2(\alpha-1)} R_{2}(p) .
$$

This fact directly follows from theorem 19 of the book [34]. It is easy to check that the second bound (22) cannot be stronger than (20). Subtracting the right-hand side of (22) from the right-hand side of (20), we obtain

$$
\frac{\alpha-2}{2(\alpha-1)}\left(2 R_{\infty}(p)-R_{2}(p)\right) \geq 0 .
$$

The latter is equivalent to $\left(\max p_{n}\right)^{2} \leq \sum_{n} p_{n}^{2}$. Taking (20), we may generally obtain better bounds. In the following, the result (20) will be used in deriving uncertainty bounds for MUMs in terms of the Rényi entropies.

\section{INDICES OF COINCIDENCE FOR MUMS}

In this section, we will study indices of coincidence for measurements considered. Let $\mathcal{P}=\left\{\mathrm{P}_{n}\right\}_{n=1}^{d}$ be a mutually unbiased measurement in $d$-dimensional Hilbert space. If the pre-measurement state is described by density matrix $\boldsymbol{\rho}$, then the probability on $n$-th outcome is written as

$$
p_{n}(\mathcal{P} \mid \boldsymbol{\rho})=\operatorname{Tr}\left(\mathrm{P}_{n} \boldsymbol{\rho}\right) .
$$

The index of coincidence is then defined as the sum of squared probabilities, namely

$$
C(\mathcal{P} \mid \boldsymbol{\rho}):=\sum_{n=1}^{d} p_{n}(\mathcal{P} \mid \boldsymbol{\rho})^{2} .
$$

It seems to be natural that the sum (25) can be linked to the quantity $\operatorname{Tr}\left(\boldsymbol{\rho}^{2}\right)$. This quantity, called the purity of $\boldsymbol{\rho}$, is frequently used since it is easy to compute [17]. The purity of a quantum state gives a good characterization of the degree of information about its preparation [35]. We have the following general bounds on (25):

$$
\frac{1}{d} \leq C\left(\mathcal{P}^{(b)} \mid \boldsymbol{\rho}\right) \leq 1
$$

Here, the lower bound follows from the convexity of the function $x \mapsto x^{2}$. The index of coincidence has been exactly calculated for a single SIC-POVM [30] and for a general SIC-POVM [31]. In both cases, resulting expression involves purity of the measured state. To derive entropic uncertainty relations, we wish to estimate from above the sum of the indices of coincidence for several mutually unbiased measurements. It is natural that our bound is formulated in terms of the purity. 
Proposition 2 Let $\mathbb{P}=\left\{\mathcal{P}^{(1)}, \ldots, \mathcal{P}^{(M)}\right\}$ be a set of $M$ mutually unbiased measurements of the efficiency $\varkappa$ in $d$ dimensions. For arbitrary $\boldsymbol{\rho}$, the sum of the corresponding indices of coincidence obey

$$
\sum_{\mathcal{P} \in \mathbb{P}} C(\mathcal{P} \mid \boldsymbol{\rho}) \leq \frac{M-1}{d}+\frac{1-\varkappa+(\varkappa d-1) \operatorname{Tr}\left(\boldsymbol{\rho}^{2}\right)}{d-1} .
$$

Proof. It follows from the construction of MUMs that any density matrix can be represented as [10]

$$
\boldsymbol{\rho}=\frac{\mathbb{1}}{d}+\sum_{b=1}^{d+1} \sum_{n=1}^{d} r_{n}^{(b)} \mathrm{F}_{n}^{(b)} .
$$

Since the operators $\mathrm{F}_{n}^{(b)}$ are all traceless and obey (6), we have

$$
\operatorname{Tr}\left(\boldsymbol{\rho}^{2}\right)=\frac{1}{d}+\sum_{b=1}^{d+1} \sum_{m, n=1}^{d} r_{m}^{(b)} r_{n}^{(b)} \operatorname{Tr}\left(\mathrm{F}_{m}^{(b)} \mathrm{F}_{n}^{(b)}\right) .
$$

By (7), we have $\operatorname{Tr}\left(\mathrm{F}_{m}^{(b)} \mathrm{F}_{n}^{(b)}\right)=-(1+\sqrt{d})^{2}$ for $m \neq n$ and

$$
\operatorname{Tr}\left(\mathrm{F}_{n}^{(b)} \mathrm{F}_{n}^{(b)}\right)=(1+\sqrt{d})^{2}(d-1) .
$$

Using these formulas, we obtain

$$
\begin{aligned}
& \sum_{m, n=1}^{d} r_{m}^{(b)} r_{n}^{(b)} \operatorname{Tr}\left(\mathrm{F}_{m}^{(b)} \mathrm{F}_{n}^{(b)}\right) \\
& =(1+\sqrt{d})^{2}(d-1) \sum_{n=1}^{d} r_{n}^{(b)} r_{n}^{(b)}-(1+\sqrt{d})^{2} \sum_{\substack{m, n=1 \\
m \neq n}}^{d} r_{m}^{(b)} r_{n}^{(b)} \\
& =(1+\sqrt{d})^{2}\left(d \sum_{n=1}^{d} r_{n}^{(b)} r_{n}^{(b)}-R^{(b)} R^{(b)}\right),
\end{aligned}
$$

where $R^{(b)}:=\sum_{n=1}^{d} r_{n}^{(b)}$. Substituting (31) into (29), we obtain an expression for $\operatorname{Tr}\left(\boldsymbol{\rho}^{2}\right)$.

Combining (8) with (28), the probability of $n$-th outcome in $b$-th measurement reads

$$
\begin{aligned}
\frac{1}{d}+t \operatorname{Tr}\left(\rho \mathrm{F}_{n}^{(b)}\right) & =\frac{1}{d}+t \sum_{m=1}^{d} r_{m}^{(b)} \operatorname{Tr}\left(\mathrm{F}_{m}^{(b)} \mathrm{F}_{n}^{(b)}\right) \\
& =\frac{1}{d}+t(1+\sqrt{d})^{2}\left(d r_{n}^{(b)}-R^{(b)}\right) .
\end{aligned}
$$

The last expression is obtained similarly to (31). Squaring this probability and further summing with respect to $n=1, \ldots, d$, one gets

$$
C\left(\mathcal{P}^{(b)} \mid \boldsymbol{\rho}\right)=\frac{1}{d}+t^{2}(1+\sqrt{d})^{4}\left(d^{2} \sum_{n=1}^{d} r_{n}^{(b)} r_{n}^{(b)}-d R^{(b)} R^{(b)}\right) .
$$

Here, we used $\sum_{n=1}^{d}\left(d r_{n}^{(b)}-R^{(b)}\right)=0$. By $(26)$, we see that, for all $b$, the second term in the right-hand side of (33) is non-negative.

Combining (29), (31), and (33), we further write

$$
\begin{aligned}
\sum_{b=1}^{M} C\left(\mathcal{P}^{(b)} \mid \boldsymbol{\rho}\right) & =\frac{M}{d}+t^{2} d(1+\sqrt{d})^{4} \sum_{b=1}^{M}\left(d \sum_{n=1}^{d} r_{n}^{(b)} r_{n}^{(b)}-R^{(b)} R^{(b)}\right) \\
& \leq \frac{M}{d}+t^{2} d(1+\sqrt{d})^{2}\left(\operatorname{Tr}\left(\boldsymbol{\rho}^{2}\right)-\frac{1}{d}\right) \\
& =\frac{M-1}{d}+\varkappa+\frac{\varkappa d-1}{d-1}\left(\operatorname{Tr}\left(\boldsymbol{\rho}^{2}\right)-1\right) .
\end{aligned}
$$


At the last step, we used (9). The quantity (34) is easily reduced to the right-hand side of (27).

The statement of Proposition 2 provides an upper bound on the sum of the indices of coincidence for a set of MUMs. For the complete set of $d+1$ MUMs, we actually have an exact result instead of inequality:

$$
\sum_{b=1}^{d+1} C\left(\mathcal{P}^{(b)} \mid \boldsymbol{\rho}\right)=1+\frac{1-\varkappa+(\varkappa d-1) \operatorname{Tr}\left(\boldsymbol{\rho}^{2}\right)}{d-1} .
$$

Indeed, the inequality (34) is saturated with $M=d+1$. For pure states, the right-hand side of (35) becomes $1+\varkappa$. This result was presented in [10] and then used in the context of entanglement detection in [36]. The inequality (27) is tight in the sense that it is always saturated with the completely mixed state $\boldsymbol{\rho}_{*}=\mathbb{1} / d$. Since operators $\mathrm{F}_{n}^{(b)}$ are all traceless, we see from (32) that

$$
p_{n}\left(\mathcal{P}^{(b)} \mid \boldsymbol{\rho}_{*}\right)=\frac{1}{d}
$$

irrespectively to $n$ and $b$. For each $b=1, \ldots, d+1$, therefore, the index of coincidence reads

$$
C\left(\mathcal{P}^{(b)} \mid \boldsymbol{\rho}_{*}\right)=\sum_{n=1}^{d} \frac{1}{d^{2}}=\frac{1}{d} .
$$

Hence, the left-hand side of (27) is equal to $M / d$ for the completely mixed state $\boldsymbol{\rho}_{*}$. By substitution $\operatorname{Tr}\left(\boldsymbol{\rho}_{*}^{2}\right)=1 / d$, the right-hand side of (27) gives $M / d$ as well. Thus, our result is almost precise for impure states with the purity close to $1 / d$. The bound (27) may also be saturated with pure states. Below, we will shortly mention an example for MUBs. Note that the purity $\operatorname{Tr}\left(\boldsymbol{\rho}^{2}\right)$ can be expressed in terms of the Bloch vector of $\boldsymbol{\rho}$ [31]. Hence, the formulas (27) and (35) can be rewritten via the Bloch vector as well. We refrain from presenting the details here.

It should be noticed that the results (27) and (35) are calculated for the aforementioned construction of mutually unbiased measurements. Setting $\varkappa=1$, nevertheless, the inequality (27) leads to the correct result for mutually unbiased bases. As was shown in [37], for a set $\mathbb{B}=\left\{\mathcal{B}^{(1)}, \ldots, \mathcal{B}^{(M)}\right\}$ of $M$ mutually unbiased bases we have

$$
\sum_{\mathcal{B} \in \mathbb{B}} C(\mathcal{B} \mid \boldsymbol{\rho}) \leq \frac{M-1}{d}+\operatorname{Tr}\left(\boldsymbol{\rho}^{2}\right) .
$$

We consider $\boldsymbol{\rho}=|\psi\rangle\langle\psi|$ with $|\psi\rangle$ taken from one of the bases $\mathcal{B}^{(1)}, \ldots, \mathcal{B}^{(M)}$. In this case, the inequality (38) is actually saturated. Indeed, one the indices $C(\mathcal{B} \mid \rho)$ is then equal to 1 and other are all $1 / d$. The sum of these indices is equal to the right-hand side of $(38)$ for $\operatorname{Tr}\left(\boldsymbol{\rho}^{2}\right)=1$. Using (38), uncertainty relations in terms of the Shannon entropies have been obtained [37]. Extensions with the use of generalized entropies were derived in [30]. The authors of [13] considered applications of the bound (38) in the context of entanglement detection. In the following, we will use (27) for obtaining entropic bounds for an arbitrary set of MUMs. We will also revisit applications of MUMs in entanglement detection.

\section{ENTROPIC UNCERTAINTY RELATIONS FOR MUMS}

In this section, we present uncertainty relations for an arbitrary set of mutually unbiased measurements. First, we obtain uncertainty relations in terms of the Rényi entropies. Second, we give formulations in terms of the Tsallis $\alpha$-entropies of order $\alpha \in(0 ; 2]$. Both the state-dependent and state-independent formulations are considered. In the Tsallis case, we also address uncertainty relations with detection inefficiencies. Our first result is posed as follows.

Proposition 3 Let $\mathbb{P}=\left\{\mathcal{P}^{(1)}, \ldots, \mathcal{P}^{(M)}\right\}$ be a set of $M$ mutually unbiased measurements of the efficiency $\varkappa$ in $d$ dimensions. For $\alpha \in[2 ; \infty]$ and arbitrary density matrix $\boldsymbol{\rho}$ on $\mathcal{H}$, the averaged sum of Rényi's entropies satisfies the state-dependent bound

$$
\begin{aligned}
\frac{1}{M} \sum_{\mathcal{P} \in \mathbb{P}} R_{\alpha}(\mathcal{P} \mid \boldsymbol{\rho}) & \geq \frac{1}{\alpha-1} \ln \left(\frac{M d(d-1)}{M(d-1)+(\varkappa d-1)\left(\operatorname{Tr}\left(\boldsymbol{\rho}^{2}\right) d-1\right)}\right) \\
& +\frac{\alpha-2}{\alpha-1}\left\{\ln d-\ln \left(1+M^{-1 / 2} \sqrt{\varkappa d-1} \sqrt{\operatorname{Tr}\left(\boldsymbol{\rho}^{2}\right) d-1}\right)\right\} .
\end{aligned}
$$


Proof. First, we will obtain a lower bound on the averaged sum of collision entropies. Since the function $x \mapsto-\ln x$ is convex, we write

$$
\frac{1}{M} \sum_{\mathcal{P} \in \mathbb{P}} R_{2}(\mathcal{P} \mid \boldsymbol{\rho})=\sum_{\mathcal{P} \in \mathbb{P}} \frac{1}{M}(-\ln C(\mathcal{P} \mid \boldsymbol{\rho})) \geq-\ln \left(\frac{1}{M} \sum_{\mathcal{P} \in \mathbb{P}} C(\mathcal{P} \mid \boldsymbol{\rho})\right) .
$$

It follows from (27) that

$$
\frac{1}{M} \sum_{\mathcal{P} \in \mathbb{P}} C(\mathcal{P} \mid \boldsymbol{\rho}) \leq \frac{M(d-1)+(\varkappa d-1)\left(\operatorname{Tr}\left(\boldsymbol{\rho}^{2}\right) d-1\right)}{M d(d-1)} .
$$

As the function $x \mapsto-\ln x$ decreases, combining (40) with (41) leads to the result

$$
\frac{1}{M} \sum_{\mathcal{P} \in \mathbb{P}} R_{2}(\mathcal{P} \mid \boldsymbol{\rho}) \geq \ln \left(\frac{M d(d-1)}{M(d-1)+(\varkappa d-1)\left(\operatorname{Tr}\left(\boldsymbol{\rho}^{2}\right) d-1\right)}\right) .
$$

The second step is to get a lower bound on the averaged sum of min-entropies. It follows from lemma 3 of [30] that

$$
\max \left\{p_{n}: 1 \leq n \leq d\right\} \leq \frac{1}{d}(1+\sqrt{d-1} \sqrt{C(p) d-1}),
$$

where $C(p)$ is the index of coincidence. For clarity, we introduce the function

$$
g_{d}(x):=\frac{1}{d}(1+\sqrt{d-1} \sqrt{x d-1}) .
$$

This function is concave and increasing. Combining these facts with (43) and (41), we obtain

$$
\begin{aligned}
& \frac{1}{M} \sum_{\mathcal{P} \in \mathbb{P}} \max _{n} p_{n}(\mathcal{P} \mid \boldsymbol{\rho}) \leq \sum_{\mathcal{P} \in \mathbb{P}} \frac{1}{M} g_{d}(C(\mathcal{P} \mid \boldsymbol{\rho})) \leq g_{d}\left(\frac{1}{M} \sum_{\mathcal{P} \in \mathbb{P}} C(\mathcal{P} \mid \boldsymbol{\rho})\right) \\
& \leq g_{d}\left(\frac{M(d-1)+(\varkappa d-1)\left(\operatorname{Tr}\left(\boldsymbol{\rho}^{2}\right) d-1\right)}{M d(d-1)}\right) .
\end{aligned}
$$

Calculating the term (45) in line with the definition (44) leads to

$$
\frac{1}{M} \sum_{\mathcal{P} \in \mathbb{P}} \max _{n} p_{n}(\mathcal{P} \mid \boldsymbol{\rho}) \leq \frac{1}{d}\left(1+M^{-1 / 2} \sqrt{\varkappa d-1} \sqrt{\operatorname{Tr}\left(\boldsymbol{\rho}^{2}\right) d-1}\right) .
$$

Due to convexity of the function $x \mapsto-\ln x$, we further write

$$
\frac{1}{M} \sum_{\mathcal{P} \in \mathbb{P}} R_{\infty}(\mathcal{P} \mid \boldsymbol{\rho}) \geq-\ln \left(\frac{1}{M} \sum_{\mathcal{P} \in \mathbb{P}} \max _{n} p_{n}(\mathcal{P} \mid \boldsymbol{\rho})\right) .
$$

Since this function decreases, combining (46) with (47) finally gives

$$
\frac{1}{M} \sum_{\mathcal{P} \in \mathbb{P}} R_{\infty}(\mathcal{P} \mid \boldsymbol{\rho}) \geq \ln d-\ln \left(1+M^{-1 / 2} \sqrt{\varkappa d-1} \sqrt{\operatorname{Tr}\left(\boldsymbol{\rho}^{2}\right) d-1}\right) .
$$

Using both the bounds (42) and (48), we complete the proof of (39) due to (20).

The statement of Proposition 3 gives a state-dependent lower bound on the sum of corresponding Rényi's entropies. In the case of MUBs, we have $\varkappa=1$. Let $\mathbb{B}=\left\{\mathcal{B}^{(1)}, \ldots, \mathcal{B}^{(M)}\right\}$ be a set of $M$ mutually unbiased bases. For $\alpha \in[2 ; \infty]$, there holds

$$
\begin{aligned}
\frac{1}{M} \sum_{\mathcal{B} \in \mathbb{B}} R_{\alpha}(\mathcal{B} \mid \boldsymbol{\rho}) & \geq \frac{1}{\alpha-1} \ln \left(\frac{M d}{M+\operatorname{Tr}\left(\boldsymbol{\rho}^{2}\right) d-1}\right) \\
& +\frac{\alpha-2}{\alpha-1}\left\{\ln d-\ln \left(1+M^{-1 / 2} \sqrt{d-1} \sqrt{\operatorname{Tr}\left(\boldsymbol{\rho}^{2}\right) d-1}\right)\right\} .
\end{aligned}
$$


For internal points of the interval $\alpha \in[2 ; \infty]$, this result gives an improvement of the uncertainty relations of [30].

The bound (39) is tight in the following sense. For arbitrary $\alpha \in[2 ; \infty]$, this inequality is certainly saturated with the completely mixed state. Substituting $\operatorname{Tr}\left(\boldsymbol{\rho}_{*}^{2}\right)=1 / d$, for $\alpha \geq 2$ the relation (39) finally gives

$$
\frac{1}{M} \sum_{\mathcal{P} \in \mathbb{P}} R_{\alpha}\left(\mathcal{P} \mid \boldsymbol{\rho}_{*}\right) \geq \frac{1}{\alpha-1} \ln d+\frac{\alpha-2}{\alpha-1} \ln d=\ln d
$$

Since the distribution (36) is uniform, we have $R_{\alpha}\left(\mathcal{P} \mid \boldsymbol{\rho}_{*}\right)=\ln d$ for all $\alpha>0$. Thus, the lower bound is actually saturated.

In the case of pure states, we obtain a state-independent lower bound. If $\boldsymbol{\rho}=|\psi\rangle\langle\psi|$, then we have Tr$\left(\boldsymbol{\rho}^{2}\right)=1$. Substituting this into the right-hand side of (39), one gets

$$
\begin{aligned}
\frac{1}{M} \sum_{\mathcal{P} \in \mathbb{P}} R_{\alpha}(\mathcal{P} \mid \psi) & \geq \frac{1}{\alpha-1} \ln \left(\frac{M d}{M+\varkappa d-1}\right) \\
& +\frac{\alpha-2}{\alpha-1}\left\{\ln d-\ln \left(1+M^{-1 / 2} \sqrt{\varkappa d-1} \sqrt{d-1}\right)\right\} .
\end{aligned}
$$

Of course, this lower bound is also valid for all mixed states. For impure $\boldsymbol{\rho}$, the lower bound (39) is stronger than (51) due to $\operatorname{Tr}\left(\boldsymbol{\rho}^{2}\right)<1$. That is, the right-hand side of (39) increases with a deviation of the purity from 1 . Dependence of such a kind seems to be natural.

The bound (39) covers the interval $\alpha \in[2 ; \infty]$. For the interval $\alpha \in(0 ; 2]$, we have a lower bound independent of $\alpha$. Recall that the Rényi entropy cannot increase with growth of $\alpha$. When $0<\alpha<2$, the formula (42) remains valid after replacing $R_{2}(\mathcal{P} \mid \boldsymbol{\rho})$ with $R_{\alpha}(\mathcal{P} \mid \boldsymbol{\rho})$. In particular, we obtain lower bounds on the sum of Shannon entropies. For a set $\mathbb{P}$ of $M$ MUMs of the efficiency $\varkappa$, we have

$$
\begin{aligned}
\frac{1}{M} \sum_{\mathcal{P} \in \mathbb{P}} H_{1}(\mathcal{P} \mid \boldsymbol{\rho}) & \geq \ln \left(\frac{M d(d-1)}{M(d-1)+(\varkappa d-1)\left(\operatorname{Tr}\left(\boldsymbol{\rho}^{2}\right) d-1\right)}\right) \\
& \geq \ln \left(\frac{M d}{M+\varkappa d-1}\right) .
\end{aligned}
$$

Substituting $M=d+1$, the latter formula gives the state-independent relation derived in [10]. Thus, we have extended this result in the following three directions: (i) our bounds hold for any set of MUMs; (ii) they are written in terms of generalized entropies; (iii) they are state-dependent. Let us proceed to the Tsallis formulation.

Proposition 4 Let $\mathbb{P}=\left\{\mathcal{P}^{(1)}, \ldots, \mathcal{P}^{(M)}\right\}$ be a set of $M$ mutually unbiased measurements of the efficiency $\varkappa$ in $d$ dimensions. For $\alpha \in(0 ; 2]$ and arbitrary density matrix $\boldsymbol{\rho}$ on $\mathcal{H}$, the averaged sum of Tsallis' entropies satisfies the state-dependent bound

$$
\frac{1}{M} \sum_{\mathcal{P} \in \mathbb{P}} H_{\alpha}(\mathcal{P} \mid \boldsymbol{\rho}) \geq \ln _{\alpha}\left(\frac{M d(d-1)}{M(d-1)+(\varkappa d-1)\left(\operatorname{Tr}\left(\boldsymbol{\rho}^{2}\right) d-1\right)}\right) .
$$

Proof. We will use the following fact. For $\alpha \in(0 ; 2]$ and arbitrary probability distribution, the Tsallis $\alpha$-entropy obeys [30]

$$
H_{\alpha}(p) \geq \ln _{\alpha}\left(\frac{1}{C(p)}\right)
$$

The inequality (54) is based on the fact that the function $x \mapsto \ln _{\alpha}(1 / x)$ is convex for $\alpha \in(0 ; 2]$. Applying Jensen's inequality to this convex function, one gets

$$
\frac{1}{M} \sum_{\mathcal{P} \in \mathbb{P}} H_{\alpha}(\mathcal{P} \mid \boldsymbol{\rho}) \geq \sum_{\mathcal{P} \in \mathbb{P}} \frac{1}{M} \ln _{\alpha}\left(\frac{1}{C(\mathcal{P} \mid \boldsymbol{\rho})}\right) \geq \ln _{\alpha}\left\{\left(\frac{1}{M} \sum_{\mathcal{P} \in \mathbb{P}} C(\mathcal{P} \mid \boldsymbol{\rho})\right)^{-1}\right\}
$$

From $x \leq y$ we have $\ln _{\alpha}(1 / x) \geq \ln _{\alpha}(1 / y)$, since the function $x \mapsto \ln _{\alpha}(1 / x)$ is decreasing. Combining (55) with (41) completes the proof. 
The statement of Proposition 4 gives a state-dependent lower bound on the average Tsallis entropy for a set of $M$ MUMs. Setting $\varkappa=1$, the bound (53) is reduced to the uncertainty relation derived in [30] for mutually unbiased bases. For a pure state $\boldsymbol{\rho}=|\psi\rangle\langle\psi|$, its purity is equal to 1 . Then the bound (53) reads

$$
\frac{1}{M} \sum_{\mathcal{P} \in \mathbb{P}} H_{\alpha}(\mathcal{P} \mid \psi) \geq \ln _{\alpha}\left(\frac{M d}{M+\varkappa d-1}\right) .
$$

It is easy to see that the state-independent lower bound (56) remain valid for all states. Similarly to (39), the right-hand side of (53) increases as the purity decreases. When $\alpha=1$, the formulas (53) and (56) also lead to (52).

Let us consider uncertainty relations with detection inefficiencies. By the parameter $\eta \in[0 ; 1]$, we characterize an efficiency of used detectors. The maximum $\eta=1$ corresponds to the inefficiency-free case. We will assume that, for any MUM, the inefficiency-free distribution is distorted according to (17). In other words, for all $\mathcal{P} \in \mathbb{P}$ we write

$$
p_{n}^{(\eta)}(\mathcal{P} \mid \boldsymbol{\rho})=\eta p_{n}(\mathcal{P} \mid \boldsymbol{\rho}), \quad p_{\varnothing}^{(\eta)}(\mathcal{P} \mid \boldsymbol{\rho})=1-\eta
$$

By $H_{\alpha}^{(\eta)}(\mathcal{P} \mid \boldsymbol{\rho})$, we mean the $\alpha$-entropy calculated for $(57)$. The theoretical value $H_{\alpha}(\mathcal{P} \mid \boldsymbol{\rho})$ is related to the case of inefficiency-free implementation of measurements. Using (18) and (53), we obtain

$$
\frac{1}{M} \sum_{\mathcal{P} \in \mathbb{P}} H_{\alpha}^{(\eta)}(\mathcal{P} \mid \boldsymbol{\rho}) \geq \eta^{\alpha} \ln _{\alpha}\left(\frac{M d(d-1)}{M(d-1)+(\varkappa d-1)\left(\operatorname{Tr}\left(\boldsymbol{\rho}^{2}\right) d-1\right)}\right)+h_{\alpha}(\eta),
$$

where $\alpha \in(0 ; 2]$ and $\mathbb{P}$ is a set of $M$ MUMs of the efficiency $\varkappa$. The result (58) is an entropic uncertainty relation in the model of detection inefficiencies. We see that the inefficiency-free bound (53) is multiplied by the factor $\eta^{\alpha}$ and also added by the binary entropy $h_{\alpha}(\eta)$. Thus, additional uncertainties are induced by non-ideal detectors [30, 31]. By setting $\alpha=1$, the relation (58) gives a lower bound on the sum of the Shannon entropies.

In their usual form, uncertainty relations are not directly applicable to study cryptographic security. To fill such a gap, entropic uncertainty relations in the presence of quantum memory should be considered [38-40]. In the case of two measurements, entropic uncertainty relations can be based on majorization techniques. This important approach has been studied in recent works [41-43]. In certain cases, the majorization approach has allowed to improve previous bounds. It would be interesting to apply majorization techniques to mutually unbiased measurement and compare resulting bounds with the above one. We hope to address this question in future investigations.

\section{ENTANGLEMENT DETECTION WITH ARBITRARY SET OF MUMS}

In this section, we address a problem of entanglement detection. Applications of mutually unbiased bases in this question were analyzed in [13]. The entanglement detection via SIC-POVMs has shortly been discussed in [30]. The use of a general SIC-POVM for such purposes was discussed in [44]. The authors of [36] extended some results of [13] with the use of mutually unbiased measurements. More separability criteria are discussed in [45, 46]. Note that the scheme of [36] is very particular in the sense that it is based on using only complete sets of MUMs. Hence, this scheme needs $d(d+1)$ local POVM elements. At the same time, the method based on a SIC-POVM uses $d^{2}$ POVM elements [44]. Here, we deal with a number of measurement operators to be performed. From the viewpoint of implementation, this number may be treated as an experimental complexity of the given scheme of entanglement detection. We will show that implementation costs of entanglement detection with MUMs can be reduced essentially.

We now consider a bipartite system of two $d$-dimensional subsystems. Its Hilbert space is the product $\mathcal{H}_{A B}=$ $\mathcal{H}_{A} \otimes \mathcal{H}_{B}$ of two isomorphic spaces $\mathcal{H}_{A}$ and $\mathcal{H}_{B}$. Let us choose the orthonormal basis $\left\{\left|i_{S}\right\rangle\right\}$, where $S=A, B$, for each of the two spaces $\mathcal{H}_{A}$ and $\mathcal{H}_{B}$. A maximally entangled pure state is then expressed as

$$
\left|\Phi_{A B}^{+}\right\rangle=\frac{1}{\sqrt{d}} \sum_{i=1}^{d}\left|i_{A}\right\rangle \otimes\left|i_{B}\right\rangle .
$$

Entangled states are a basic resource in quantum information science. Hence, the problem of efficient detection of entanglement is of great importance [7, 47].

Let us recall shortly basic notions. A product state is any state of the form $\boldsymbol{\rho}_{A} \otimes \boldsymbol{\rho}_{B}[17]$. When both the matrices $\boldsymbol{\rho}_{A}$ and $\boldsymbol{\rho}_{B}$ are rank-one, we have a pure product state. A bipartite mixed state is called separable, when its density matrix $\widetilde{\boldsymbol{\rho}}_{A B}$ can be represented as a convex combination of product states [45]. That is, there exist a probability distribution $\left\{q_{k}\right\}$ and two sets $\left\{\boldsymbol{\rho}_{A}^{(k)}\right\}$ and $\left\{\boldsymbol{\rho}_{B}^{(k)}\right\}$ such that

$$
\tilde{\boldsymbol{\rho}}_{A B}=\sum_{k} q_{k} \boldsymbol{\rho}_{A}^{(k)} \otimes \boldsymbol{\rho}_{B}^{(k)} .
$$


Note that each separable state can also be expressed as a convex combination of only pure product states. This fact easily follows from (60) by substitution of the corresponding spectral decompositions. When representations of the form (60) is not possible, the state is called entangled [45].

To detect entanglement, we aim to use a collection of local measurements. Let $\mathbb{P}_{A}=\left\{\mathcal{P}_{A}^{(1)}, \ldots, \mathcal{P}_{A}^{(M)}\right\}$ and $\mathbb{Q}_{B}=$ $\left\{\mathcal{Q}_{B}^{(1)}, \ldots, \mathcal{Q}_{B}^{(M)}\right\}$ be two sets of $M$ MUMs. In $b$-th joint measurement, the pair $(m, n)$ of local outcomes occurs with the probability

$$
P^{(b)}(m, n)=\operatorname{Tr}\left(\left(\mathrm{P}_{m}^{(b)} \otimes \mathrm{Q}_{n}^{(b)}\right) \widetilde{\boldsymbol{\rho}}_{A B}\right) .
$$

Following the idea of [13], we introduce a quantity

$$
J_{M}\left(\widetilde{\boldsymbol{\rho}}_{A B}\right)=\sum_{b=1}^{M} \sum_{n=1}^{d} P^{(b)}(n, n) .
$$

In the case of mutually unbiased bases, this correlation measure was proposed in [13]. The definition (62) is an immediate extension to MUMs.

Proposition 5 Let $\mathbb{P}_{A}$ be a set of $M$ mutually unbiased measurements of the efficiency $\varkappa_{A}$ in the d-dimensional space $\mathcal{H}_{A}$. Let $\mathbb{Q}_{B}$ be a set of $M$ mutually unbiased measurements of the efficiency $\varkappa_{B}$ in the d-dimensional space $\mathcal{H}_{B}$. For all density matrices $\boldsymbol{\rho}_{A} \in \mathcal{L}_{+}\left(\mathcal{H}_{A}\right)$ and $\boldsymbol{\rho}_{B} \in \mathcal{L}_{+}\left(\mathcal{H}_{B}\right)$, the correlation measure satisfies

$$
J_{M}\left(\boldsymbol{\rho}_{A} \otimes \boldsymbol{\rho}_{B}\right) \leq \prod_{S=A, B}\left(\frac{M(d-1)+\left(\varkappa_{S} d-1\right)\left(\operatorname{Tr}\left(\boldsymbol{\rho}_{S}^{2}\right) d-1\right)}{d(d-1)}\right)^{1 / 2} .
$$

Proof. For a product state $\widetilde{\boldsymbol{\rho}}_{A B}=\boldsymbol{\rho}_{A} \otimes \boldsymbol{\rho}_{B}$, we clearly have

$$
P^{(b)}(n, n)=p_{n}\left(\mathcal{P}_{A}^{(b)} \mid \boldsymbol{\rho}_{A}\right) p_{n}\left(\mathcal{Q}_{B}^{(b)} \mid \boldsymbol{\rho}_{B}\right) .
$$

Using equation (64) and the Cauchy-Schwarz inequality, we then obtain

$$
\begin{aligned}
J_{M}\left(\boldsymbol{\rho}_{A} \otimes \boldsymbol{\rho}_{B}\right) & =\sum_{b=1}^{M} \sum_{n=1}^{d} p_{n}\left(\mathcal{P}_{A}^{(b)} \mid \boldsymbol{\rho}_{A}\right) p_{n}\left(\mathcal{Q}_{B}^{(b)} \mid \boldsymbol{\rho}_{B}\right) \\
& \leq\left(\sum_{b=1}^{M} C\left(\mathcal{P}_{A}^{(b)} \mid \boldsymbol{\rho}_{A}\right)\right)^{1 / 2}\left(\sum_{b=1}^{M} C\left(\mathcal{Q}_{B}^{(b)} \mid \boldsymbol{\rho}_{B}\right)\right)^{1 / 2}
\end{aligned}
$$

Combining the latter with (27) completes the proof.

The statement of Proposition 5 leads to a necessary criterion that the given bipartite state is a product state. Applying this criterion for the given input $\widetilde{\boldsymbol{\rho}}_{A B}$, we should treat $\boldsymbol{\rho}_{A}$ and $\boldsymbol{\rho}_{B}$ as the reduced density matrices. As usual, they are obtained by the partial trace operation:

$$
\boldsymbol{\rho}_{A}=\operatorname{Tr}_{B}\left(\widetilde{\boldsymbol{\rho}}_{A B}\right), \quad \boldsymbol{\rho}_{B}=\operatorname{Tr}_{A}\left(\widetilde{\boldsymbol{\rho}}_{A B}\right) .
$$

Relations between some norms of operators before and after partial trace with applications to quantum entropies were obtained in [48]. To use the formula (63), we need only purities of these density matrices. Substituting the purities in the right-hand side of (63), we should then compare the result with the actual value $J_{M}\left(\widetilde{\boldsymbol{\rho}}_{A B}\right)$. This value is calculated from the measurement statistics. If the condition (63) is violated, then the input $\widetilde{\boldsymbol{\rho}}_{A B}$ is certainly not a product state.

It is of interest to adopt (63) for the case, when purities of the reduced density matrices are unknown. If we keep at least purity of the state $\widetilde{\boldsymbol{\rho}}_{A B}$ per se, then a state-dependent form of the criterion can still be given. If the input $\tilde{\boldsymbol{\rho}}_{A B}$ is a product state then

$$
\operatorname{Tr}\left(\boldsymbol{\rho}_{A}^{2}\right) \operatorname{Tr}\left(\boldsymbol{\rho}_{B}^{2}\right)=\operatorname{Tr}\left(\widetilde{\boldsymbol{\rho}}_{A B}^{2}\right), \quad \operatorname{Tr}\left(\boldsymbol{\rho}_{A}^{2}\right)+\operatorname{Tr}\left(\boldsymbol{\rho}_{B}^{2}\right) \leq 1+\operatorname{Tr}\left(\widetilde{\boldsymbol{\rho}}_{A B}^{2}\right) .
$$

The second relation is proved as follows. If real numbers $x, y \in[0 ; 1]$ are connected as $x y=a$, then $x+y \leq 1+a$, by convexity of the function $x \mapsto x+a / x$. Combining (63) with (67) leads to the following statement. If the given density matrix $\widetilde{\boldsymbol{\rho}}_{A B}$ is a product then

$$
J_{M}\left(\widetilde{\boldsymbol{\rho}}_{A B}\right) \leq \frac{1}{d(d-1)} \sqrt{\Gamma^{2}+(\varkappa d-1)\left[\Gamma d+(M+\varkappa d-1)(d-1) d \operatorname{Tr}\left(\widetilde{\boldsymbol{\rho}}_{A B}^{2}\right)\right]},
$$


where $\Gamma=M(d-1)-(\varkappa d-1)$. The formula $(68)$ gives a necessary criterion, which depends on purity of the tested bipartite state. As the violation of (68) has been observed, we truly conclude that the tested state is not a product state. Finally, we present the following state-independent bound.

Proposition 6 Let $\mathbb{P}_{A}$ be a set of $M$ mutually unbiased measurements of the efficiency $\varkappa_{A}$ in the $d$-dimensional space $\mathcal{H}_{A}$. Let $\mathbb{Q}_{B}$ be a set of $M$ mutually unbiased measurements of the efficiency $\varkappa_{B}$ in the d-dimensional space $\mathcal{H}_{B}$. If the given state $\widetilde{\boldsymbol{\rho}}_{A B}$ is separable then

$$
J_{M}\left(\widetilde{\boldsymbol{\rho}}_{A B}\right) \leq \frac{1}{d} \sqrt{M+\varkappa_{A} d-1} \sqrt{M+\varkappa_{B} d-1}
$$

Proof. We first observe the following. For any product state, the quantity $J_{M}\left(\boldsymbol{\rho}_{A} \otimes \boldsymbol{\rho}_{B}\right)$ is bounded from above by the right-hand side of (69). This claim directly follows from $(63)$ and $\operatorname{Tr}\left(\boldsymbol{\rho}_{S}^{2}\right) \leq 1$. As the function $x \mapsto x^{2}$ is convex, we further have

$$
J_{M}\left(\widetilde{\boldsymbol{\rho}}_{A B}\right) \leq \sum_{k} q_{k} J_{M}\left(\boldsymbol{\rho}_{A}^{(k)} \otimes \boldsymbol{\rho}_{B}^{(k)}\right)
$$

Combining (70) with the above observation completes the proof due to the condition $\sum_{k} q_{k}=1$.

The statement of Proposition 6 gives a necessary criterion for separability of bipartite states. In the case $\varkappa_{A}=$ $\varkappa_{B}=\varkappa$, this criterion reads

$$
J_{M}\left(\widetilde{\boldsymbol{\rho}}_{A B}\right) \leq \frac{M+\varkappa d-1}{d}
$$

With $M=d+1$, we deal with the scheme using two complete sets of MUMs. Then the formula (71) is reduced to $J_{M}\left(\widetilde{\boldsymbol{\rho}}_{A B}\right) \leq 1+\varkappa$. The latter necessary criterion for the separability was discussed in [36]. We have seen that entanglement detection may be proceeded with a lesser number of measurements. This approach could be easy for implementation. On the other hand, a sensitivity of the scheme will probably decrease. In the following, we give a reason for trade-off between a sensitivity of the scheme and its experimental complexity.

In general, the above upper bounds give only a necessary criterion for the separability of bipartite states. For some classes of states, however, this criterion may be sufficient as well. We shall now illustrate this fact with isotropic states. Recall that isotropic states are states of the form

$$
\widetilde{\boldsymbol{\rho}}_{\text {iso }}=\gamma\left|\Phi_{A B}^{+}\right\rangle\left\langle\Phi_{A B}^{+}\right|+(1-\gamma) \widetilde{\boldsymbol{\rho}}_{A B *} .
$$

Here, $\gamma \in[0 ; 1]$ and $\widetilde{\boldsymbol{\rho}}_{A B *}$ is the completely mixed state on $\mathcal{H}_{A} \otimes \mathcal{H}_{B}$, namely

$$
\widetilde{\boldsymbol{\rho}}_{A B *}=\frac{\mathbb{1}_{A} \otimes \mathbb{1}_{B}}{d^{2}}
$$

To the given MUM $\mathcal{P}_{A}^{(b)}=\left\{\mathrm{P}_{n}\right\}$ of the efficiency $\varkappa$, we assign a set $\mathcal{P}_{B}^{(b) *}=\left\{\mathrm{P}_{n}^{*}\right\}$ of operators that are conjugate in the following sense. For all $i, j=1, \ldots, d$, matrix elements obey

$$
\left\langle i_{B}\left|\mathrm{P}_{n}^{*}\right| j_{B}\right\rangle=\left\langle j_{A}\left|\mathrm{P}_{n}\right| i_{A}\right\rangle
$$

It is easy to check that the set $\mathcal{P}_{B}^{(b) *}$ is also a MUM of the efficiency $\varkappa$. Using the property $(74)$, we obtain

$$
\left\langle\Phi_{A B}^{+}\left|\mathrm{P}_{n} \otimes \mathrm{P}_{n}^{*}\right| \Phi_{A B}^{+}\right\rangle=\frac{1}{d} \operatorname{Tr}\left(\mathrm{P}_{n} \mathrm{P}_{n}\right)=\frac{\varkappa}{d}
$$

For the isotropic state (72), calculations then lead to the result

$$
J_{M}\left(\widetilde{\boldsymbol{\rho}}_{i s o}\right)=M\left(\gamma \varkappa+\frac{1-\gamma}{d}\right) .
$$

For the value $M=d+1$, this result was presented in [36]. Using the scheme with $M$ MUMs, we can certainly detect entanglement for those values $\gamma$ that satisfy

$$
\frac{M+\varkappa d-1}{M d}<\gamma \varkappa+\frac{1-\gamma}{d},
$$

or merely $\gamma>1 / M$. Thus, schemes with two sets of $M$ mutually unbiased measurements allow to detect the entanglement of all isotropic states with $\gamma \in(1 / M ; 1]$. For schemes with mutually unbiased bases, this result was 
discussed in [13]. In this sense, there is a good reason to realize entanglement detection with MUMs instead of MUBs. The interval $\gamma \in(1 / M ; 1]$ widens with growth of $M$. Here, we see some trade-offs between a sensitivity of the considered scheme and costs for its implementation. The maximal interval is obtained for $M=d+1$. It is known that isotropic states are certainly entangled for $\gamma>1 /(d+1)$ [47]. Hence, using two complete sets of MUMs allows to detect all the entanglement of isotropic states [36].

Note that the described scheme of entanglement detection can be realized for arbitrary $d$. Indeed, the explicit construction for $d+1$ mutually unbiased measurements has been presented in [10]. However, acceptable values of $\varkappa$ cannot be chosen a priori. In this way, therefore, we cannot generally obtain a set of $d+1$ mutually unbiased bases. The problem of maximal set of mutually unbiased bases seems to be very hard. In practical questions, however, we may try to adopt MUMs instead of MUBs. This possibility was already discussed in [10, 36]. The above results also support such an approach to applications of mutual unbiasedness in quantum information processing.

\section{CONCLUSIONS}

We have studied some properties of recently introduced mutually unbiased measurements. For a set of several MUMs, we estimated from above the sum of corresponding indices of coincidence. The presented results are essentially based on such calculations. Then we have obtained entropic uncertainty relations for a set of several MUMs in terms of the Rényi and Tsallis entropies. The derived relations include both the state-dependent and state-independent forms. The state-dependent bound on the sum of corresponding Rényi's entropies turns to be tight in some sense. Namely, for all orders this bound is saturated with the completely mixed state. We also obtained some improvement of the Rényi entropic bounds for mutually unbiased bases. The Tsallis formulation allowed to address uncertainty relations with detection inefficiencies. Applications of mutually unbiased measurements in entanglement detection were considered in more details. In particular, we obtained results for an arbitrary number of MUMs used in entanglement detection. It seems that there is a certain trade-off between a sensitivity of the scheme and costs for its experimental implementation. In the literature, mutually unbiased bases were considered as a suitable tool in quantum state reconstruction, quantum error correction, and the mean king's problem. It would be interesting to study possible applications of MUMs in these questions. In this regard, the results presented here may also be of significance.

Note added. After this work was completed I learned about very recent results of Chen and Fei [49]. These authors independently studied uncertainty relations for MUMs in terms of the Rényi and Tsallis entropies. The uncertainty relations presented in my work differ in the following two respects. First, an arbitrary number of MUMs was considered, whereas the authors of [49] deal with $d+1$ MUMs. Second, for $M=d+1$ the lower bound (39) is stronger than the corresponding bound of [49].

The author acknowledges fruitful discussions with Zbigniew Puchała and Karol Życzkowski in Institute of Physics, Jagiellonian University, Kraków.

[1] W. Heisenberg, Zeitschrift für Physik 43, 172-198 (1927); the translation is reprinted in Quantum Theory and Measurement, 62-84, J. A. Wheeler and W. H. Zurek eds., Princeton University Press, Princeton 1983.

[2] E. Schrödinger, Naturwissenschaften 23, 807-812, 823-828, 844-849 (1935); the translation is reprinted in Quantum Theory and Measurement, 152-167, J. A. Wheeler and W. H. Zurek eds., Princeton University Press, Princeton 1983.

[3] M. A. Nielsen and I. L. Chuang, Quantum Computation and Quantum Information, Cambridge University Press, Cambridge 2000.

[4] P. Busch, T. Heinonen, and P. J. Lahti, Phys. Rep. 452, 155-176 (2007).

[5] I. Białynicki-Birula and E. Rudnicki, Entropic uncertainty relations in quantum physics, in Statistical Complexity, 1-34, K. D. Sen ed., Springer, Berlin 2011.

[6] S. Wehner and A. Winter, New J. Phys. 12, 025009 (2010).

[7] R. Horodecki, P. Horodecki, M. Horodecki, and K. Horodecki, Rev. Mod. Phys. 81, 865-942 (2009).

[8] T. Durt, B.-G. Englert, I. Bengtsson, and K. Życzkowski, Int. J. Quantum Inf. 8, 535-640 (2010).

[9] W. K. Wootters and B. D. Fields, Ann. Phys. 191, 363-381 (1989).

[10] A. Kalev and G. Gour, New J. Phys. 16, 053038 (2014).

[11] D. Gottesman, Phys. Rev. A 54, 1862-1868 (1996).

[12] A. R. Calderbank, E. M. Rains, P. W. Shor, and N. J. A. Sloane, Phys. Rev. Lett. 78 405-408 (1997).

[13] C. Spengler, M. Huber, S. Brierley, T. Adaktylos, and B. C. Hiesmayr, Phys. Rev. A 86, 022311 (2012).

[14] L. Vaidman, Y. Aharonov, and D. Z. Albert, Phys. Rev. Lett. 58, 1385-1387 (1987).

[15] B.-G. Englert and Y. Aharonov, Phys. Lett. A 284, 1-5 (2001). 
[16] J. Watrous, Theory of Quantum Information, University of Waterloo, Waterloo 2011. http://www.cs.uwaterloo.ca/ ${ }^{\text {watrous/CS766/ }}$

[17] I. Bengtsson and K. Życzkowski, Geometry of Quantum States: An Introduction to Quantum Entanglement, Cambridge University Press, Cambridge 2006.

[18] A. Rényi, On Measures of Entropy and Information, in Proceedings of 4th Berkeley Symposium on Mathematical Statistics and Probability, J. Neyman ed., 547-561, University of California Press, Berkeley-Los Angeles 1961.

[19] K. Życzkowski, Open Sys. Inf. Dyn. 10, 297-310 (2003); corrigendum in the e-print version arXiv:quant-ph/0305062v2.

[20] H. Y. N. Ng, M. Berta, and S. Wehner, Phys. Rev. A 86, 042315 (2012).

[21] P. Mandayam, S. Wehner, and N. Balachandran, J. Math. Phys. 51, 082201 (2010).

[22] G. M. Bosyk, M. Portesi, F. Holik, and A. Plastino, Phys. Scr. 87, 065002 (2013).

[23] W. Roga, Z. Puchała, Ł. Rudnicki, and K. Życzkowski, Phys. Rev. A 87, 032308 (2013).

[24] A. E. Rastegin, J. Phys. A: Math. Theor. 46, 285301 (2013).

[25] M. Gell-Mann and C. Tsallis eds., Nonextensive Entropy - Interdisciplinary Applications, Oxford University Press, Oxford 2004.

[26] C. Tsallis, J. Stat. Phys. 52, 479-487 (1988).

[27] J. Havrda and F. Charvát, Kybernetika 3, 30-35 (1967).

[28] S. Furuichi, J. Math. Phys. 47, 023302 (2006).

[29] A. E. Rastegin, Kybernetika 48, 242-253 (2012).

[30] A. E. Rastegin, Eur. Phys. J. D 67269 (2013).

[31] A. E. Rastegin, Phys. Scr. 89, 085101 (2014).

[32] R. Chaves and T. Fritz, Phys. Rev. A 85, 032113 (2012).

[33] A. E. Rastegin, Quantum Inf. Comput. 14, 0996-1013 (2014).

[34] G. H. Hardy, J. E. Littlewood, and G. Polya, Inequalities, Cambridge University Press, London 1934.

[35] G. Adesso, A. Serafini, and F. Illuminati, Open Sys. Inf. Dyn. 12, 189-205 (2005)

[36] B. Chen, T. Ma, and S.-M. Fei, Phys. Rev. A 89, 064302 (2014).

[37] S. Wu, S. Yu, and K. Mølmer, Phys. Rev. A 79, 022104 (2009).

[38] M. Berta, M. Cristandl, R. Colbeck, J. M. Renes, and R. Renner, Nat. Phys. 6, 659-662 (2010).

[39] P. J. Coles, R. Colbeck, L. Yu, and M. Zwolak, Phys. Rev. Lett. 108, 210405 (2012).

[40] P. J. Coles and M. Piani, Phys. Rev. A 89, 022112 (2014).

[41] Z. Puchała, E. Rudnicki, and K. Życzkowski, J. Phys. A: Math. Theor. 46, 272002 (2013).

[42] S. Friedland, V. Gheorghiu, and G. Gour, Phys. Rev. Lett. 111, 230401 (2013).

[43] Ł. Rudnicki, Z. Puchała, and K. Życzkowski, Phys. Rev. A 89, 052115 (2014).

[44] B. Chen, T. Ma, and S.-M. Fei, arXiv:1406.7820v1 [quant-ph] (2014).

[45] K. Życzkowski, P. Horodecki, A. Sanpera, and M. Lewenstein, Phys. Rev. A 58, 883-892 (1998).

[46] H. Zhao, S.-M. Fei, J. Fan, and Z.-X. Wang, Int. J. Quantum Inf. 12, 1450013 (2014).

[47] M. Horodecki and P. Horodecki, Phys. Rev. A 59, 4206-4216 (1999).

[48] A. E. Rastegin, J. Stat. Phys. 148, 1040-1053 (2012).

[49] B. Chen and S.-M. Fei, arXiv:1407.6816v1 [quant-ph] (2014). 